\title{
ATLAS Micro-Pattern Gaseous Detectors production status
}

\section{Marco Schioppa*†}

INFN and Università della Calabria

E-mail: marco.schioppa@cern.ch

MICRO MEsh GAseous Structure (MM) chambers are Micro-Pattern Gaseous Detectors designed to provide a high spatial resolution and reasonable good time resolution in highly irradiated environments. This detector was chosen by the ATLAS collaboration as tracking detectors for the upgrade of the first station of the forward Muon Spectrometer. To meet the demanding performance requirements of the ATLAS Muon Spectrometer, MM chambers are required to achieve a single plane resolution better than $100 \mu \mathrm{m}$ with an efficiency better than $95 \%$ for tracks up to an inclination of $32 \mathrm{deg}$ in a magnetic field up to $0.3 \mathrm{~T}$.

A thorough test program on MM full size prototypes is just bring to an end and the assembly of $128 \mathrm{MM}$ modules to be produced and certified for the New Small Wheel (NSW) of ATLAS experiment is started. The tests performed on the first module- 0 produced excellent results, proving that the prototype fully meet the performance requirements.

The replacement in LS3 of the Inner Detector by the Inner Tracker (ITk) extends the pseudorapidity coverage for tracking up to $|\eta|<4$.0. As a consequence, it becomes interesting to identify muons in this pseudo-rapidity region. This can be accomplished installing micro-pattern gaseous or silicon pixel detectors between the endcap calorimeters and the shielding disks in front of the NSW in the region $2.7<\eta<4$. These detectors could be used to identify (tag) ITk tracks as muons, relying entirely on the ITk for the momentum measurement. There are currently different proposals for the instrumentation of this region.

The ATLAS NSW MM assembly methodologies, the related quality controls and the candidate technologies for the $\eta$-tagger are presented in this document.

5th International Conference on Micro-Pattern Gas Detectors (MPGD2017)

22-26 May, 2017

Philadelphia, USA

${ }^{*}$ Speaker.
${ }^{\dagger}$ On behalf of ATLAS Collaboration 


\section{Introduction}

MICRO MEsh GAseous Structure (MM or Micromegas), is an innovative design concept for Micro-Pattern Gaseous Detectors (MPGD), first introduced during the 1990s [1]. In 2007 an ambitious long-term R\&D activity was started in the context of the ATLAS experiment, at CERN: the Muon ATLAS Micromegas Activity (MAMMA). After years of tests on prototypes and technology breakthroughs, this high-resolution, radiation-hard detector has been chosen as the main tracking technology for the upgrade of the innermost station of the high rapidity region (named Small Wheel) of the ATLAS [4] Muon Spectrometer. The upgrade is necessary to take advantage from the improved LHC operation in the third run of the LHC $2-3 \cdot 10^{34} \mathrm{~cm}^{-2} \mathrm{~s}^{-1}$ and of the HighLuminosity LHC (HL-LHC) [5], where the instantaneous luminosity of the collider is foreseen to reach up to $7 \cdot 10^{34} \mathrm{~cm}^{-2} \mathrm{~s}^{-1}$.

The Small Wheel will be replaced by a new system (New Small Wheel) [2] during the second long shutdown of the LHC. This new system is composed by Micromegas and small-strip Thin Gap Chambers (sTGC) both able to work at high rates (up to $15 \mathrm{kHz} / \mathrm{cm}^{2}$ ) with good spatial and time resolution. They will be capable both to guarantee a muon momentum resolution at level of $15 \% P_{T}$ for muon of $1 \mathrm{TeV} / \mathrm{c}$ momentum and to keep single muon trigger under control. Figure 1 shows three possible track constellations which lead at present to an accepted muon track in this region. Combining the information of the New Small Wheel and the Big Wheel a clear distinction between a muon coming from the interaction point and background hits will be possible. For this an angular resolution better than 1 mrad is necessary in the NSW. The MM detector, thanks to its

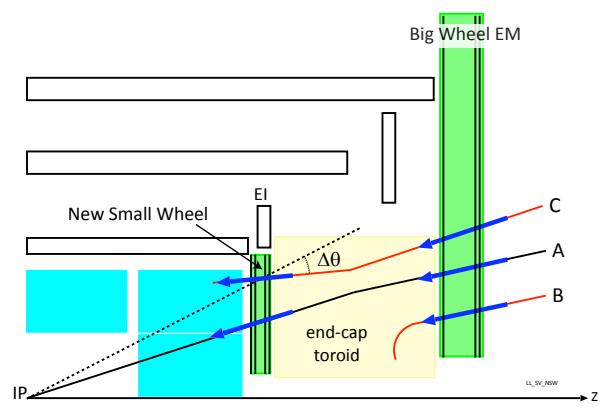

Figure 1: Schematic view of a quarter of the ATLAS detector with possible tracks in the muon end-cap region. The existing Big Wheel trigger accepts all three tracks shown. With the NSW enhancement of the muon end-cap trigger only track A, the desired track, which is confirmed by both the Big Wheel and the New Small Wheel, will be accepted. Track B gives only a signal in the Big Wheel and Track C does not point towards the interaction point. The NSW logic requires that $\Delta \theta< \pm 7 \mathrm{mrad}$.

very fine read-out strip segmentation, together with a reasonably good time resolution, can also be exploited to complement the trigger scheme based on STGC, adding robustness and redundancy to the system.

Moreover the muon spectrometer of the ATLAS detector will know another significant upgrade during the third long LHC shutdown (2023-2024) in order to cope with the operational conditions at the HL-LHC in the fourth run and beyond [3]. A high- $\eta$ tagger is under consideration to extend the angular acceptance for muon identification up to $|\eta|<4$. 


\section{The New Small Wheel}

Figure 2 shows the layout of the two sides of each NSW detector. Each wheel is composed of 16 sectors, 8 large and 8 small. Each sector will have modules of sTGC and of MM detectors, arranged in the order sTGC-MM-MM-sTGC, each module being a quadruplet of detector layers. Therefore, 16 points will be measured for each track in the NSW layout. MM will be mainly used for tracking, while sTGC will be mainly exploited for the trigger system. An overall active area of about $1200 \mathrm{~m}^{2}$ will be provided by each one of the two technologies employed in the Muon Spectrometer. Both technologies will be used for tracking and for the trigger, therefore ensuring a redundant and flexible detector system. A crucial goal of the NSW is to improve the precision
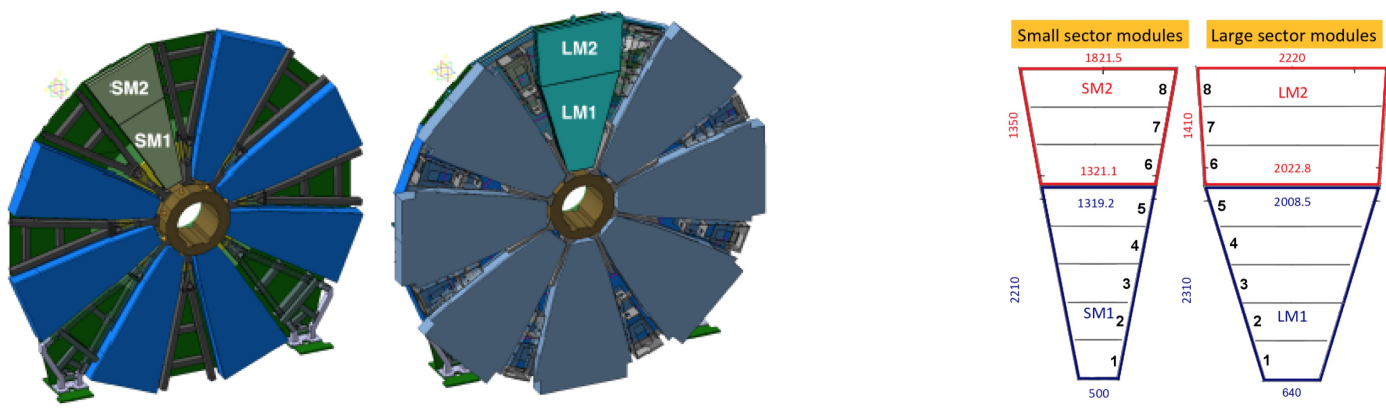

Figure 2: Left: view of the two sides of each NSW detector. Right: Micromegas chambers segmentation. Four types of chambers are in construction: SM1 and SM2 form the small sectors while LM1 and LM2 form the large ones.

of the current muon trigger system in ATLAS. The increase of the radiation background expected for the 3rd and 4th LHC runs will inevitably lead to a higher rate of trigger signals produced by fake tracks. An increase of momentum threshold would ensure a reduction of the trigger rate to an acceptable level but would cause a loss of efficiency for physics events. To maintain the same efficiency with a trigger rate within the acceptable bandwidth, the NSW will provide a tighter selection of tracks pointing to the interaction vertex. This will significantly reduce the trigger rate produced by forward tracks in the muon spectrometer, as shown in Figure 1.

\section{Working principle of resistive strips Micromegas detector}

The working principle of MM chambers, with the layout chosen for the ATLAS NSW, is shown in Figure 3. MM are parallel-plate chambers where the amplification takes place in a thin gap, separated from the conversion region $5 \mathrm{~mm}$ wide by a thin stainless steel micro-mesh ( 325 lines/inch), supported by $128 \mu \mathrm{m}$ high insulating pillars with a pitch of few millimeters. The detector is filled with a $\mathrm{Ar}: \mathrm{CO}_{2}$ (93:7) gas mixture. The cathode electrode is at about $-300 \mathrm{~V}$ with respect to the mesh, which is grounded. This potential produces an electric field capable to leads ionisation electrons, produced by ionising particles, towards the mesh. Here the electric field lines take the funnel shape helping the electrons to enter in the very thin amplification region between the mesh and the readout electrode, where a $540-580 \mathrm{~V}$ voltage is applied. A factor $E_{A} / E_{D} \approx 70-100$ is required for full mesh transparency for electrons. The thin amplification region enables fast evacuation of ions, which occurs approximately in $100 \mathrm{~ns}$, allowing the MM detector to operate in highly irradiated 


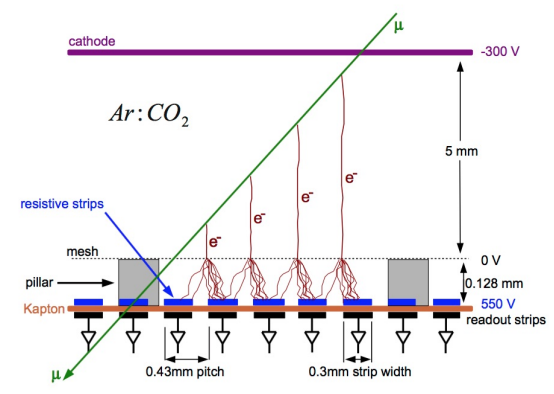

Figure 3: Sketch of charged particle detection using a Micromegas detector. A muon (green line) traverses the detector ionizing the $\mathrm{Ar}: \mathrm{CO}_{2}$ (93:7) gas. The electrons drift to the mesh and cause avalanches between the mesh and the anode strips. The accumulated charge is coupled capacitively to the readout strips underneath the anode strips.

environments. Due to the very high electric field in the amplification region the drifting electrons produce avalanches, with a gain of the order of $10^{4}$. The signal produced by these avalanches is then read by readout strips. With this high electric field discharges can occur between mesh and strips when the charge density in this region exceeds the Raether limit [6]. This causes a temporarily reduced amplification field and thus dead time. In 2007 the R\&D program MAMMA between Atlas Collaboration, RD51 Collaboration (MPGD) and Cern, started to solve this problem and define the optimal MM detector layout for the ATLAS NSW detector. To prevent this global voltage drop a layer of resistive strips $\approx 10 \mathrm{M} \Omega \cdot \mathrm{cm}^{-1}$ is added on top of the readout electrode [7] [8]. In this way the discharge affects only few strips. For electronic readout the charge collected on the resistive strips is capacitively coupled to the readout strips. To achieve the required muon position precision the MM strips have $300 \mu m$ width and $425 \mu m$ (450 $\mu m$ at low $\eta$ ) pitch. With these thin readout strips, by measuring the time of arrival of the signal on them and knowing the drift velocity of electrons in the gas mixture, the position of the primary ionisation electrons can be reconstructed for each strip, allowing for track reconstruction in a single detector plane. With typical drift velocities of $50 \mu \mathrm{m} / \mathrm{ns}$ (better if saturated) electrons need $100 \mathrm{~ns}$ for a $5 \mathrm{~mm}$ gap and spatial resolution of about $100 \mu \mathrm{m}$ or better can be achieved.

\section{ATLAS New Small Wheel detector layout}

The NSW have the identical segmented structure of the current Small Wheel: eight alternating small and large sectors. Each MM sector is subdivided into two sub-sectors of trapezoidal shape as shown in Figure 2-left. There are four different Micromegas modules with different dimensions as illustrated in Figure 2-right, which are built and assembled in four different construction sites. The modules SM1 are built in Italy, SM2 in Germany, LM1 in France and LM2 in Russia and Greece. The unprecedented size of these modules represents an important challenge in the development of the construction technique. In fact to achieve the requirements of muon reconstruction in the NSW, it is required a precision on the alignment of the strips on each detection layer of $30 \mu \mathrm{m} \mathrm{rms} \mathrm{in} \eta$ and $80 \mu \mathrm{m}$ rms in $z$ and a flatness of each panel less than $37 \mu \mathrm{m}$ rms $(110 \mu \mathrm{m}$ max deviation with respect to the nominal plane).

To reach an optimal track resolution each module consists of eight consecutive MM detectors, 
which are formed by two quadruplets. Each quadruplet is formed by five sandwich panels. Figure 4 shows a schematic cross section of a quadruplet: closed (left) and exploded (right). One readout
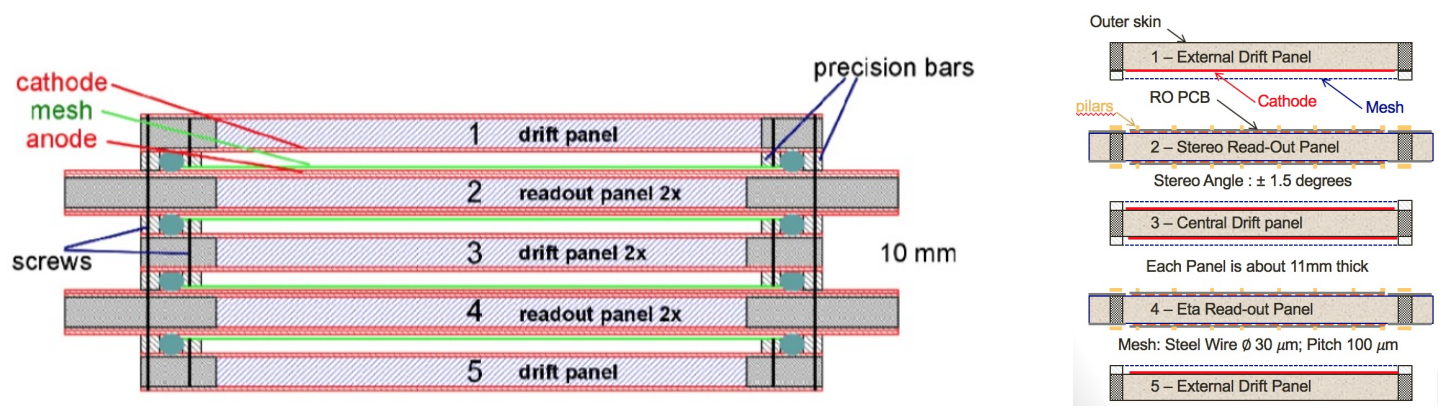

Figure 4: Left: scheme of the five sandwich panels assembled to a quadruplet: two external drift panels, one central drift panel, one double $\eta$ readout panel and one double stereo readout panel. The four active gas volumes between the drift and readout panels are defined by extruded precision drift bars $(5.20 \mathrm{~mm})$. Similarly, extruded mesh bars define the exact distance of the micro-meshes from the cathodes $(5.06 \mathrm{~mm})$. Right: exploded view of a MM module. The stainless steel mesh (30 $\mu \mathrm{m}$ wire diameter and $100 \mu \mathrm{m}$ pitch) is glued on the mesh bars which in turn is glued onto the copper side of the drift panel (cathode).

panel of a quadruplet has on both sides a strip configuration parallel to the upper and lower bound (named $\eta$-strips). The other readout panel has stereo strips tilted by $+1.5^{\circ}$ (named s-strips) on one side and $-1.5^{\circ}$ (named s'-strips) on the other side with respect to the $\eta$ strips. The stereo strips deliver a rough information about the azimuthal $\phi$-coordinate of the muon. Between the double quadruplet structure a spacer frame will be installed for stable mounting.

\section{MM panels construction}

In order to reach the desired muon position resolution of better than $100 \mu \mathrm{m}$ in a single detector layer, the panels are sandwiches of $0.5 \mathrm{~mm}$ thick FR4 PCB sheets with aluminum honeycomb in the middle and aluminum frames in the perimeter and in the joint of two adjacent PCBs. The total thickness of the panels is $\approx 11 \mathrm{~mm}$. The construction sites of the four different modules use similar methods for the panel assembling to reach the mechanical requirements. Individual adaptions are accounted for by locally available infrastructure and manpower. The common idea is to transfer the planarity of a very planar surface (e.g. a granite table) to PCBs by application of vacuum. A frame of extruded aluminum bars and a honeycomb structure are glued on top of the PCB positioned on the reference surface. In the so called 'vacuum bag technique' this structure is covered with a plastic bag and sucked down again the granite table until glue curing. After that this structure is turned up side down and glued over the second layer of PCBs placed onto the granite table, covered again with a plastic bag and sucked down to the granite table to complete the panel. In the 'stiff-back technique' the second layer of PCBs, sucked down again to a second planar surface, is glued at fixed and precise height, simultaneously or in a second step, to the 'PCB-aluminum framehoneycomb' structure. As glue the two component epoxy adhesive ARALDITE 2011 is used to assemble all parts of the panels. Its curing time is about 24 hours.

The method to build the readout and drift panels is similar. The readout ones need very sophisticated alignment tools to guarantee the necessary precision on strip positioning. Several masks 
present on the board guide the board alignment into the panel. Here, each surface sheet of a panel consists of three or five PCBs, depending on the module, since single readout sheets in the full size are not available. The readout boards have maximum dimension of $45 \times 200 \mathrm{~cm}^{2}$ with 1022 strips/board. Figures 5 show the structure of the readout board (left) and the first readout panel of SM1 module zero built in Pavia (right).
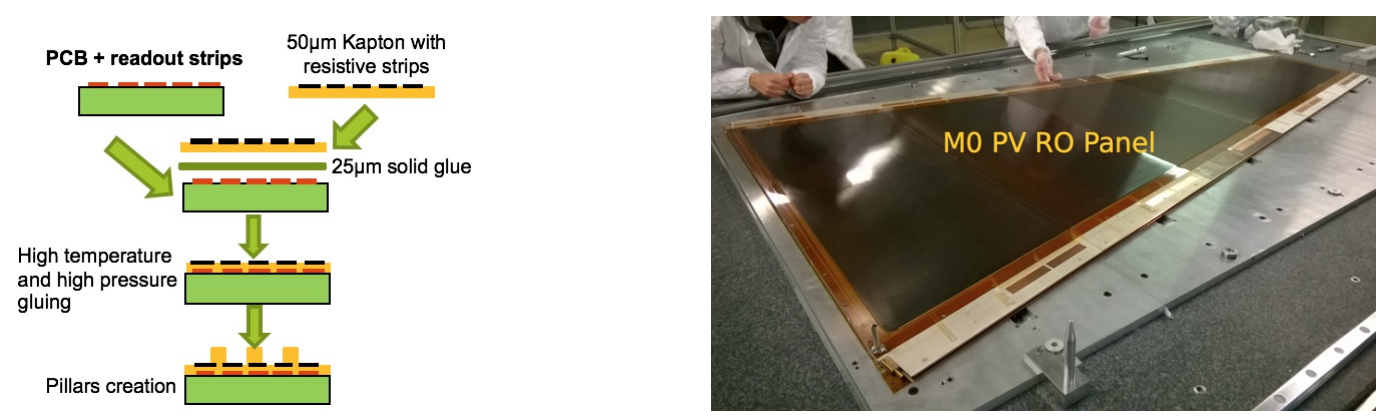

Figure 5: Left: the readout board is made of FR4 PCB with cooper strips coupled with $50 \mu m$ Kapton foils with resistive strips by means of $25 \mu \mathrm{m}$ thin film of solid glue. By high temperature and high pressure the two foils stick together. On top of the resistive strips is glued a Pyralux coverlay from which a regular structure of pillars are made lithographically. Right: readout panel for SM1 module zero.

The drift panels are built with the same planarity and thickness requirements of the readout panel. In addition a stainless steel micro-mesh with wires of $28 \mu \mathrm{m}$ diameter and $100 \mu \mathrm{m}$ pitch is glued onto $5.06 \mathrm{~mm}$ thick extruded bars which are in turn glued and screwed on the FR4 surface of the cathode sides of the drift panels. These bars follow the drift panel shape as shown in the Figure 6left and provide the correct distance between mesh and the cathode surface. The mesh will thus be attached to ground potential. This so called floating mesh construction has the advantage that the amplification regions are still accessible even after the mounting of the meshes, an important feature for these large area detectors. Before glueing the meshes all drift panels must be gas leak tested and all meshes and panels must be dust and dirt free. The meshes will be stretched with a homogeneous tension of $\approx 10 \mathrm{~N} \cdot \mathrm{cm}^{-1}$ using pneumatic clamps, as shown in Figure 6-right, and glued to transfer frames. The additional glueing provides gas tightness between the active detector volume and the honeycomb volume. The mesh is then glued to the mesh frame and the transfer frame can be reused after cleaning for the next mesh. For the gas tightness an O-ring is used in the groove between the mesh frame and the outer drift frame. The latter defines the distance between anode and cathode and thus the drift region.

\section{MM module assembly}

The constructed panels for a module are first cleaned and then assembled together in a clean room. Figure 7-left shows the vertical assembly scheme. Here the outer drift panels are reinforced by stiff frames which are removed after the assembly. The alignment of $\eta$ and stereo readout panels is ensured by two alignment pins glued into the stereo readout panel. Figure 7-right shows the assembled SM1 module zero with the stiff frames still attached. 

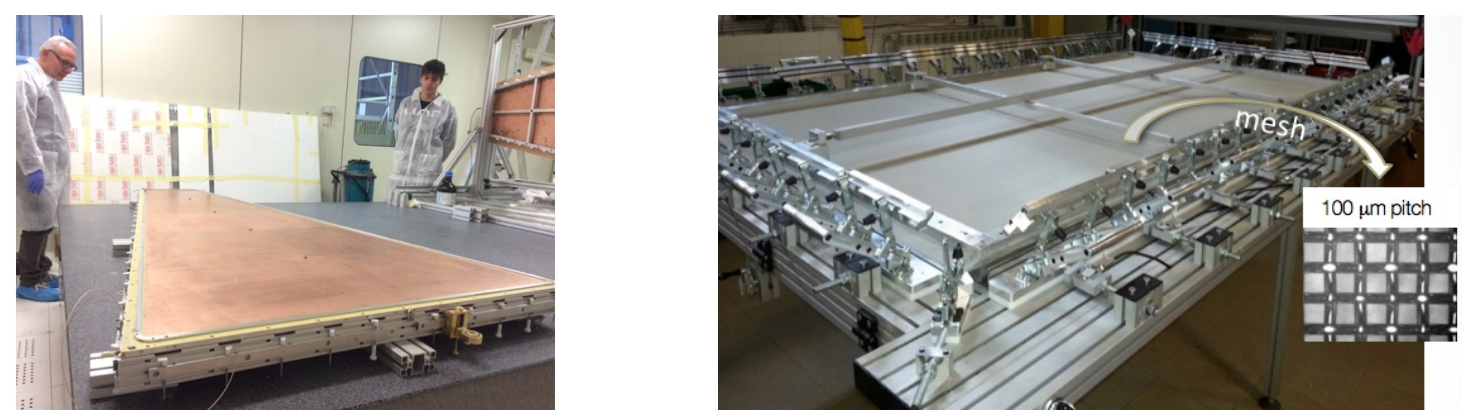

Figure 6: Left: the drift panel with the extruded mesh bars glued ready to be finalized with mesh. Right: stretching of the mesh.
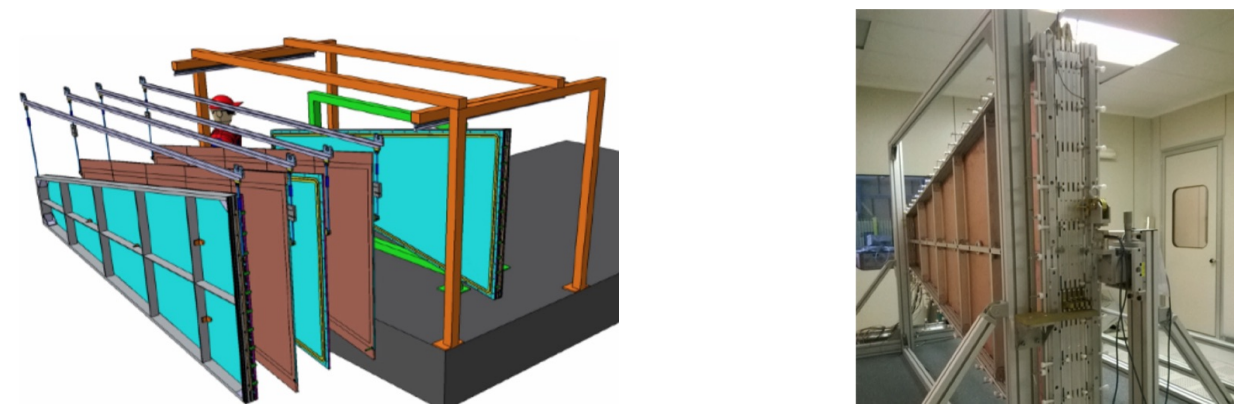

Figure 7: Left: assembly scheme of the quadruplet in vertical mounting structure. Vertical mounting in a clean room reduces the influence of dust and dirt. Right: the SM1 module zero.

\section{Module zero test beam results}

The module- 0 was exposed to a particle beam to verify the performances of the produced prototype respect to the project requirements. The measurements were performed at CERN in June 2016 at the $\mathrm{H} 4$ beam line of the SPS, using a $180 \mathrm{GeV} / \mathrm{c} \pi^{+}$beam with rate $1-500 \mathrm{kHz}$ and beam spot of about $1 \times 1 \mathrm{~cm}^{2}$. The chamber was filled with a $\mathrm{Ar}: \mathrm{CO}_{2}(93: 7)$ gas mixture at atmospheric pressure with a flux rate of $20 \mathrm{l} / \mathrm{h}$. The experimental setup was composed by a detectors array of which the module-0 is a part as shown in Figure 8-left.

In order to measure the spatial resolution of two SM1 layers, the residuals have been evaluated both for $\eta$ and $\phi$ coordinate. The residual distributions have been fitted with a gaussian function. Data collected on PCB5 (longest strips) have been used for both coordinates, with tracks almost orthogonal to the chamber, which allow for the centroid method to be used for reconstruction. The spatial resolution resulted $81 \mu \mathrm{m}$ for eta and $2.4 \mathrm{~mm}$ for $\phi$ coordinate. Figure 8-right shows the residual distribution for the $\eta$ coordinate.

A possible misalignment of the readout strips is an independent source of resolution error and is not controlled by the previous checks, which rely on a local measurement. Therefore a determination of the displacement of the strips in the $\eta$ coordinate as a function of the $\phi$ coordinate has been performed for the different layers of PCB5 and PCB3. This kind of measurement could be an indication of layer-to-layer rotation or strip pattern global deformation. The measurement is performed by aligning the first layer with respect to tracks reconstructed in the reference chambers and then looking at the average residual reconstructed in the other layers as a function of the second 
coordinate $\mathrm{Y}$. The quoted precision of the $\mathrm{Y}$ coordinates corresponds to the scanned area with the beam. The displacements reported for both PCB5 and PCB3 are within $\pm 80 \mu m$, consistent with the requirements.

The homogeneity and the efficiency of the Module- 0 response have also been studied. To calculate the software efficiency, a fiducial region that take into account cut selections, has been defined. The software efficiency is the ratio $N_{i n} / N_{\text {tot }}$, where $N_{\text {in }}$ is the number of events with at least one cluster within $1.5 \mathrm{~mm}$ from the extrapolated position and $N_{t o t}$ is the number of events that pass the cut selections in the fiducial region. The efficiency at nominal HV values is $96 \%$ approximately equal to all layers, as expected for this detector.

The module zero of SM2 has been tested and shows similar results.
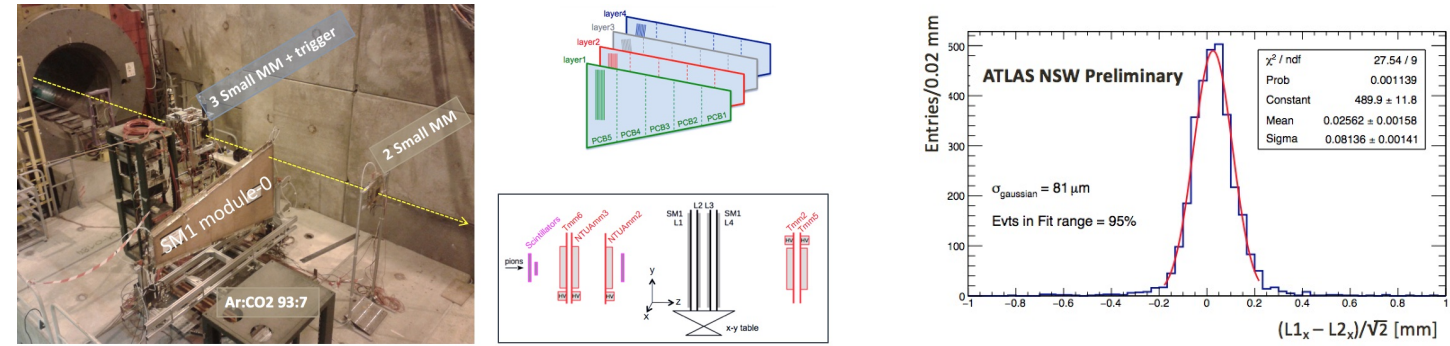

Figure 8: Left: SM1 module zero during the test beam at Cern in the H8 experimental area. Central top the scheme of readout strips. Central bottom the test beam setup. Right the residual distribution of the $\eta$ coordinate.

\section{ATLAS MM mass production}

The construction and test of the four different types of MM quadruplets, 2 for small (SM1, SM2) and 2 for large sectors (LM1, LM2), is done in institutes, distributed over several countries. For each type 32 chambers have to be built (overall total: 128). Figure 9 shows a collage of pictures relative to the activities in all the construction sites. All the activities are well advanced. The full size module- 0 has been built in all construction sites and two of them have built the second one (SM1 and LM2). In parallel the production of drift and readout panels is starting in all construction sites.

\section{Phase II $\eta$-tagger}

The ATLAS Phase-II upgrade concludes the process of adapting the muon spectrometer to the ever increasing performance of the LHC. A high- $\eta$ tagger is under consideration to extend the angular acceptance for muon identification. It is less advanced than the rest of the muon upgrade program and requires further $R \& D$ work before a technical design can be presented.The aim of this project is to tag muons in the region $2.7<\eta<4$ where the expected particle rate reaches $10 \mathrm{MHz} / \mathrm{cm}^{2}$. The tagger is located after the forward calorimeter as shown in Figure 10. It could be used to tag tracks reconstructed by ITk as muons, relying entirely on the ITk for the momentum measurement. There are currently different proposals for the instrumentation of this region, like 

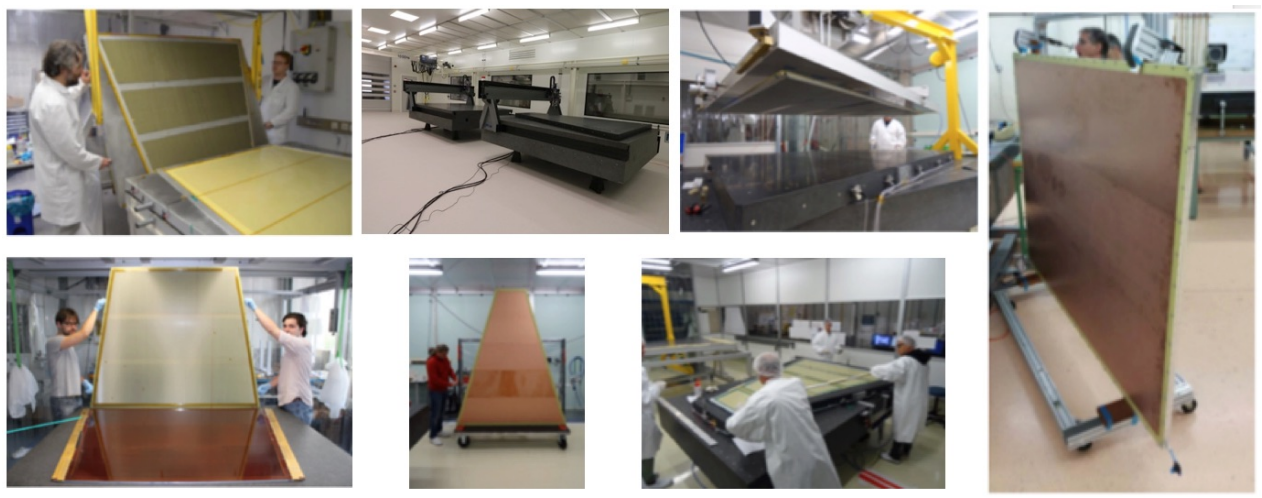

Figure 9: Collage of pictures relative to the construction phases in all the sites involved in the MM NSW project.

Pixelated MM [9], Precision Timing [10], $\mu$-RWELL [11] and $\mu$-PIC [12]. All these technologies have very low and are suitable technology for this purpose.
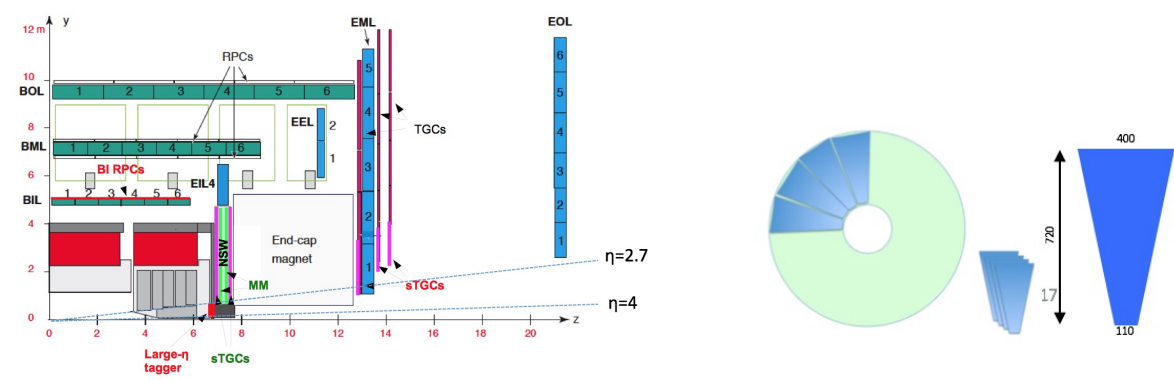

$\mathrm{R}_{\mathrm{ext}}=93 \mathrm{~cm}$

$\mathrm{R}_{\text {int }}=25 \mathrm{~cm}$

Thickness $=5 \mathrm{~cm}$

$z=6800 m m$

Figure 10: Left: quarter of the ATLAS detector whit indicated the location of $\eta$ tagger. Right: segmentation and dimensions of this detector.

\section{Conclusions}

The design and construction for series production of high-rate capable large size Micromegas chambers for the upgrade of the ATLAS muon spectrometer is here described. To cope with the required precision on muon tracking position (e.g. readout strip alignment within $30 \mu \mathrm{m}$ in $\eta$ direction) special assembly methods and tools had been developed as well as a complete set of quality controls on the elements and the final modules. These methods have been tested and refined during the construction of the full size prototypes (module zeros) in all construction sites. The first test beam executed at CERN with $180 \mathrm{GeV}$ pions beam on SM1 module zero shows very satisfactory results. All sites has started the series production and, despite a very tight schedule, the plan is to be ready for installation during the second LHC long shutdown that is within 2020. Finally the muon $\eta$-tagger seems worthwhile for ATLAS but many studies still needed to decide the technologies to be adopted.

\section{Acknowledgment}

The author would like to thank all the colleagues involved in the Muon ATLAS NSW 
Micromegas upgrade for the huge work done in the past years and under way to complete this project, which has been only partially reviewed in this paper, and for the full support provided during the preparation of the talk presented at the MPGD 2017 conference and of this report.

\section{References}

[1] Giomataris, Y. et al., Micromegas: A High granularity position sensitive gaseous detector for high particle flux environments, NIM A376 (1996) 29-35

[2] Kawamoto, T. et al., ATLAS New Small Wheel Technical Design Report, CERN- LHCC-2013-006; ATLAS-TDR-020-2013

[3] ATLAS Collaboration, Technical Design Report for the Phase-II Upgrade of the ATLAS Muon Spectrometer, CERN-LHCC-2017-017; ATLAS-TDR-026

[4] ATLAS Collaboration, The ATLAS Experiment at the CERN Large Hadron Collider, JINST 3 (2008) S08003

[5] Lucio Rossi and Oliver Bruning, Final Evaluation of the Baseline and Most Probable Alternatives : Milestone: MS23, CERN-ACC-2015-0017

[6] H. Raether, Electron Avalanches and Breakdown in Gases Butterworths (1964)

[7] Burnens, J. et al., A spark-resistant bulk-Micromegas chamber for high- rate applications, CERN-PH-EP-2010-061

[8] T. Alexopoulos et al., A spark-resistant bulk-Micromegas chamber for high-rate applications, NIM A 640 (2011) $110-118$

[9] M. Lupberger, et al., Pixelated Micromegas detectors for a pixel-TPC, PoS TIPP2014 (2014) 225

[10] T. Papaevangelou, A picosecond Micromegas EUV photodetector, 8th symposium on large TPCs, Paris 5-7/12/16

[11] G. Bencivenni et al., The micro-Resistive WELL detector: a compact spark-protected single amplification-stage MPGD, JINST 10 P02008

[12] A. Ochi et al., Development of a Micro Pixel Chamber for the ATLAS Upgrade, Physics Procedia Volume 37, 2012, Pages 554-560 